OPEN ACCESS

Edited by:

Edmund C. Lalor,

Trinity College Dublin, Ireland

Reviewed by:

Kevin M. Spencer,

VA Boston Healthcare

System/Harvard Medical School, USA

Brian F. O'Donnell,

Indiana University, USA

*Correspondence:

Julian Keil

julian.keil@charite.de

Received: 23 November 2015 Accepted: 25 February 2016

Published: 14 March 2016

Citation:

Keil J, Roa Romero Y, Balz J, Henjes M and Senkowski D (2016) Positive and Negative Symptoms in Schizophrenia Relate to Distinct Oscillatory Signatures of Sensory

Gating.

Front. Hum. Neurosci. 10:104. doi: 10.3389/fnhum.2016.00104

\section{Positive and Negative Symptoms in Schizophrenia Relate to Distinct Oscillatory Signatures of Sensory Gating}

\author{
Julian Keil*, Yadira Roa Romero, Johanna Balz, Melissa Henjes and Daniel Senkowski \\ Multisensory Integration Group, Department of Psychiatry and Psychotherapy, St. Hedwig Hospital, Charité - \\ Universitätsmedizin Berlin, Berlin, Germany
}

Oscillatory activity in neural populations and temporal synchronization within these populations are important mechanisms contributing to perception and cognition. In schizophrenia, perception and cognition are impaired. Aberrant gating of irrelevant sensory information, which has been related to altered oscillatory neural activity, presumably contributes to these impairments. However, the link between schizophrenia symptoms and sensory gating deficits, as reflected in oscillatory activity, is not clear. In this electroencephalography study, we used a paired-stimulus paradigm to investigate frequency-resolved oscillatory activity in 22 schizophrenia patients and 22 healthy controls. We found sensory gating deficits in patients compared to controls, as reflected in reduced gamma-band power and alpha-band phase synchrony difference between the first and the second auditory stimulus. We correlated these markers of neural activity with a five-factor model of the Positive and Negative Syndrome Scale. Gamma-band power sensory gating was positively correlated with positive symptoms. Moreover, alpha-band phase synchrony sensory gating was negatively correlated with negative symptoms. A cluster analysis revealed three schizophrenia phenotypes, characterized by (i) aberrant gamma-band power and high positive symptoms, (ii) aberrant alphaband phase synchrony, low positive, and low negative symptom scores or (iii) by intact sensory gating and high negative symptoms. Our study demonstrates that aberrant neural synchronization, as reflected in gamma-band power and alpha-band phase synchrony, relates to the schizophrenia psychopathology. Different schizophrenia phenotypes express distinct levels of positive and negative symptoms as well as varying degrees of aberrant oscillatory neural activity. Identifying the individual phenotype might improve therapeutic interventions in schizophrenia.

Keywords: inter-trial coherence, power, gamma-band, alpha-band, phenotype, cluster analysis 


\section{INTRODUCTION}

A consistent observation in schizophrenia is that affected individuals are impaired in their ability to gate irrelevant sensory input (Patterson et al., 2008). It has been proposed that sensory gating deficits lead to a sensory overload, which contributes to cognitive deficits in schizophrenia (McGhie and Chapman, 1961; Shakow, 1963; Freedman et al., 1991; Bob et al., 2014). A commonly used experimental paradigm to study sensory gating is the auditory paired-stimulus paradigm, in which two identical stimuli are presented in close succession (Adler et al., 1982; Bramon et al., 2004). Electroencephalography (EEG) and magnetoencephalography (MEG) studies revealed that in healthy individuals the early cortical response to the second stimulus is considerably smaller than the response to the first stimulus (Clementz et al., 1997b; Hall et al., 2011; Popov et al., 2011). In schizophrenia patients, the response difference between the first and second stimulus is typically reduced (Patterson et al., 2008).

Surprisingly, only few studies have linked the sensory gating deficit in electrophysiological data to positive or negative symptoms in schizophrenia (Potter et al., 2005; Keshavan et al., 2008). Auditory gating deficits, as reflected in the P50 event-related potential (ERP), were found to be correlated with negative symptoms (Ringel et al., 2004; Louchart-de la Chapelle et al., 2005; Thoma et al., 2005). Moreover, the P50 auditory gating deficit has been correlated with the severity of auditory hallucinations (Smith et al., 2013). Measuring schizotypy in healthy individuals, a recent study revealed that the P50 auditory gating deficit relates to cognitive disorganization and impulsive non-conformity (Park et al., 2015). Taken together, these studies suggest a link between sensory gating deficits and the schizophrenia psychopathology.

In recent years, the focus of electrophysiological research has changed from the analysis of ERPs to a more complex view encompassing dynamics in cortical networks (Lisman, 2012; Uhlhaas and Singer, 2015). Numerous studies provided evidence that coordinated oscillatory activity in neural populations plays a role for cognitive processes (Singer, 1999; Senkowski et al., 2008; Uhlhaas and Singer, 2010). Moreover, aberrant oscillatory activity presumably contributes to the psychopathology of schizophrenia (Andreasen et al., 1999; Andreasen, 2000; Senkowski and Gallinat, 2015). Various studies reported deficits in oscillatory activity in this patient group (Leicht et al., 2010; Mathiak et al., 2011; Popov et al., 2011; see Lee et al., 2003a and Senkowski and Gallinat, 2015 for reviews). Interestingly, it has been hypothesized that different aspects of oscillatory activity are relevant for different symptoms in schizophrenia (Pittman-Polletta et al., 2015; Uhlhaas and Singer, 2015). Aberrant temporal synchronization might primarily contribute to cognitive impairments and negative symptoms (Andreasen et al., 1999; Andreasen, 2000). Although the evidence is less robust, negative symptoms have also been associated with reduced resting-state alpha-band power and increased low-frequency power (Sponheim et al., 2000; see Boutros et al., 2014 for a review). Furthermore, deficits in higher frequency oscillations might predominantly contribute to positive symptoms (Senkowski and Gallinat, 2015; Uhlhaas and Singer, 2015). However, results on resting-state as well as stimulus-evoked gamma-band activity are mixed (e.g., Gallinat et al., 2004; Brenner et al., 2009; Hong et al., 2012). Taken together, electrophysiological markers promise to provide important information for the identification of specific phenotypes of schizophrenia (Smucny et al., 2013; Ethridge et al., 2015).

Analyzing frequency-resolved oscillatory activity allows disentangling the influence of local synchrony, i.e., oscillatory power, from temporal synchronization, i.e., phase consistency over time. Hence, the analysis of oscillatory activity can provides important information on aberrant stimulus processing in schizophrenia (Brockhaus-Dumke et al., 2008). A number of studies suggested that stimulus-processing deficits in schizophrenia are reflected in amplitude and phase of various frequency bands (Jansen et al., 2004; Moran et al., 2012; Carolus et al., 2014). For instance, some studies reported a reduction in low-frequency power in the theta-band and alpha-band in sensory gating paradigms (Moran et al., 2012; Carolus et al., 2014). In addition to alterations in power, phase coherence in the same frequency ranges appears to be impaired in schizophrenia (Jansen et al., 2004; BrockhausDumke et al., 2008). Thus far, however, only few studies have related time-frequency resolved cortical activity to the schizophrenia symptomatology. One study revealed a reduction in evoked gamma-band activity that was associated with altered auditory and visual perception (Johannesen et al., 2008). Another study found that reduced stimulus evoked beta-band power in sensory gating was related to negative symptoms in schizophrenia (Smucny et al., 2013). Furthermore, another study showed that reduced alpha-band power in sensory gating was associated with reduced overall level of functioning and reduced processing speed (Hong et al., 2012). To summarize, recent findings suggest that reduced power and phase coherence reflect sensory gating deficits in schizophrenia.

In the present study, we investigated the relationships between positive and negative symptoms in schizophrenia and sensory gating deficits as reflected in oscillatory activity. Previously, auditory sensory processing has been linked to gamma-band activity (Pantev et al., 1991; Tiitinen et al., 1993). Moreover, there is evidence that gamma-band activity plays a role in auditory hallucinations (Reulbach et al., 2007; Spencer et al., 2009). Therefore, we hypothesized that gamma-band power in auditory gating relates to positive symptoms in schizophrenia. Aberrant temporal synchronization has been shown to affect information processing (Andreasen et al., 1999; Koh et al., 2011). Therefore, we hypothesized a relationship between phase coherence in auditory gating and negative symptoms in schizophrenia. Finally, we predicted that individual symptom patterns would enable us to identify patient clusters that share common phenotypes. Exploring possible subgroups within schizophrenia could further our understanding of the underlying psychopathology (Boutros et al., 2014). 


\section{MATERIALS AND METHODS}

\section{Participants}

Twenty-two schizophrenia patients (ScZ, seven female, $37.23 \pm$ 7.75 years) with the DSM-4 diagnosis schizophrenia were recruited from outpatient units of the Charite - Universitätsmedizin Berlin. The psychiatric diagnosis was assessed by a senior psychiatrist at the recruiting institution. Twenty-two education, handedness, gender, and age matched healthy control participants (HC, eight female, $37.09 \pm 8.47$ years), who were screened for psychopathology with the German version of the Structured Clinical Interview for DSM-4-R Non-Patient Edition (SCID), were recruited from the general population (Table 1). In all participants the Brief Assessment of Cognition in Schizophrenia (BACS) was assessed (Keefe, 2004). Severity of symptoms was obtained by the Positive and Negative Syndrome Scale by trained clinicians (PANSS; Kay et al., 1987). In accordance with a 5 -factor model, items were grouped into factors "positive," "negative," "depression," "excitement," and "disorganization" (Wallwork et al., 2012). All participants gave written informed consent, had normal hearing and normal or corrected to normal vision, and no record of neurological disorders. No participant met DSM-4-R criteria for alcohol or substance abuse. A random sample of $45 \%$ of participants underwent a multi drug-screening test. The study was performed in accordance with the Declaration of Helsinki and the local ethics commission approved the study.

\section{Experimental Design}

One hundred S1-S2 stimulus pairs of $0.003 \mathrm{~s}$ white noise were presented with a $0.5 \mathrm{~s}$ onset-to-onset interstimulus interval. Stimulus pairs were presented with a random intertrial interval of 7-9 s (mean $=8 \mathrm{~s})$ at $65 \mathrm{~dB}(\mathrm{SPL})$ via a single mono speaker (Bose Companion 2) in a sound-attenuating, electrically shielded chamber. Participants were instructed to attend to auditory stimuli and were asked to keep their gaze on a small fixation cross, which was shown on a display placed in front of them.

\section{EEG Recording and Data Analysis}

Data were recorded using a 128 channel active EEG system (EasyCap, Herrsching, Germany), which included two EOG electrodes (online: $1000 \mathrm{~Hz}$ sampling rate with a 0.016$250 \mathrm{~Hz}$ bandpass filter; offline: downsampling to $500 \mathrm{~Hz}, 1-$ $125 \mathrm{~Hz}$ FIR bandpass filtering and 49.1-50.2 Hz, fourth order Butterworth notch filtering). To correct for EOG and ECG artifacts, independent component (IC) analyses were conducted (Lee et al., 1999). On average $12.12 \pm 3.80$ ICs for ScZ and $13.95 \pm 4.73$ ICs for HC were rejected based on visual inspection (Chaumon et al., 2015). Remaining noisy channels were interpolated using spherical interpolation $(\mathrm{ScZ}=15.14 \pm 4.29$ channels; $\mathrm{HC}=13.55 \pm 5.08$ channels). Analysis of EEG data focused on the comparison of oscillatory responses to paired auditory stimuli. Additionally, we analyzed the auditory evoked P50 component (see Supplementary Material). Data were rereferenced to common average and split into trials of $-1 \mathrm{~s}$ to $3 \mathrm{~s}$ around the $\mathrm{S} 1$ onset and those containing muscular artifacts or amplitudes of $\pm 100 \mu \mathrm{V}$ were rejected by visual inspection. On average, 59.32 trials for ScZ and 60.68 trials for HC were used for the further analyses. To estimate oscillatory power and temporal synchronization, single-trial data were transformed into the time-frequency domain using a multitaper approach (Mitra and Pesaran, 1999). Spectral estimates were calculated on sliding time-frequency windows $(8-80 \mathrm{~Hz}$, window size $=3$ cycles per frequency, $\pm 3.5 \mathrm{~Hz}$ frequency smoothing) based on a discrete prolate spheroid sequence of tapers with a step size of $0.025 \mathrm{~s}$ and $2 \mathrm{~Hz}$. Single-trial power was normalized to reflect the relative change from baseline ( $-0.5 \mathrm{~s}$ to $-0.1 \mathrm{~s}$ baseline window). As a measure of temporal synchronization, phase coherence across trials, i.e., inter-trial coherence (ITC), was computed on single-trial Fourier values from the same time-frequency transformation without baseline correction (Cheron et al., 2007). EEG data analysis was performed using EEGLab (Delorme and Makeig, 2004) and FieldTrip (Oostenveld et al., 2011).

\section{Statistical Analysis}

Transient auditory stimulation typically induces a mediocentral increase in oscillatory activity between 0.05 and $0.1 \mathrm{~s}$ after stimulus onset (Clementz et al., 1997b; Leicht et al., 2010; Popov et al., 2011). Therefore, we extracted $0.05 \mathrm{~s}$ intervals starting $0.05 \mathrm{~s}$ after the onset of the S1 and S2 from the power and ITC time-frequency data. In a first step, we computed sensory gating within each participant. As a measure of normalized sensory gating for single-trial power, we computed dependent-samples $t$-tests for each time-frequency tile for S2 versus $\mathrm{S} 1$ within each participant. ITC is computed across trials; therefore we used the S2-S1 difference as a measure of sensory gating. In a second step, power and ITC sensory gating values in whole frequency range of $8-80 \mathrm{~Hz}$ without á priori restriction to frequency bands were compared between groups. To this end, independentsamples $t$-tests with Monte-Carlo randomization and clusterbased correction for multiple comparisons were used (Maris and Oostenveld, 2007). This approach allows the identification of clusters of significant effects in three-dimensional space (i.e., time, frequency, and sensor), effectively controlling for multiple comparisons. To elucidate, whether ScZ and HC show different oscillatory activity following $\mathrm{S} 1$ and $\mathrm{S} 2$, additional repeated measures ANOVAs with the factors Group (ScZ vs. HC) and Stimulus (S1 vs. S2), as well as appropriate post hoc $t$-tests were computed (see Supplementary Material). Pearson correlations were computed between psychopathology scores (i.e., PANSS), oscillatory power, and ITC. S2-S1 sensory gating measures in power and ITC were tested for normality using ShapiroWilk tests. In the next step, oscillatory power and ITC were averaged over the clusters obtained in the group analysis. One patient was identified as an outlier $(>2.5 \mathrm{SD})$ based on the PANSS positive factor and was therefore excluded from further analysis. Since PANSS symptom scores were grouped into five factors, a Bonferroni-corrected alpha-level of $0.05 / 5=0.01$ was used for the two-tailed correlation between psychopathology scores and EEG data. To statistically control for the influence of antipsychotic medication, medication dosage was converted to chlorpromazine equivalent level (Gardner et al., 2010), and entered as covariate to partial correlation analyses in ScZ. 
TABLE 1 | Overview of demographic data.

\begin{tabular}{|c|c|c|c|c|c|c|}
\hline & \multicolumn{2}{|c|}{$\mathrm{ScZ}$} & \multicolumn{2}{|c|}{$\mathrm{HC}$} & \multicolumn{2}{|c|}{ Statistics } \\
\hline & Mean & $S D$ & Mean & $S D$ & $t$-values & $p$-values \\
\hline Age (years) & 37.23 & 7.75 & 37.09 & 8.47 & 0.06 & 0.96 \\
\hline Education (years) & 11.00 & 1.72 & 11.09 & 1.6 & -0.18 & 0.86 \\
\hline Daily cigarettes & 4.27 & 3.41 & 2.72 & 3.58 & 1.46 & 0.15 \\
\hline Illness duration (years) & 9.18 & 5.03 & - & - & - & - \\
\hline \multirow[t]{2}{*}{ Chlorpromazine eq. } & 387.70 & 200.45 & - & - & - & - \\
\hline & $N$ & & $N$ & & & \\
\hline Gender (m/f) & $15 / 7$ & & $14 / 8$ & & & \\
\hline Handedness (r/l) & $19 / 3$ & & $20 / 2$ & & & \\
\hline Antipsychotic med. & 22 & & - & & & \\
\hline Haloperidol & 1 & & - & & & \\
\hline Amisulpride & 8 & & - & & & \\
\hline Clozapine & 4 & & - & & & \\
\hline Quetiapine & 2 & & - & & & \\
\hline Olanzapine & 6 & & - & & & \\
\hline Aripiprazole & 3 & & - & & & \\
\hline Risperidone & 4 & & - & & & \\
\hline Paliperidone & 1 & & - & & & \\
\hline Antidepressive med. & 4 & & - & & & \\
\hline Mirtazapine & 1 & & - & & & \\
\hline Escitalopram & 2 & & - & & & \\
\hline \multirow[t]{2}{*}{ Paroxetine } & 1 & & - & & & \\
\hline & Mean & $S D$ & Mean & $S D$ & $t$-values & $p$-values \\
\hline \multicolumn{7}{|l|}{ BACS } \\
\hline Verbal memory & 42.90 & 12.63 & 48.32 & 10.80 & -1.527 & 0.134 \\
\hline Digit & 19.50 & 4.23 & 20.90 & 3.74 & -0.171 & 0.248 \\
\hline Motor & 67.18 & 11.42 & 76.59 & 10.68 & -2.822 & 0.007 \\
\hline Fluency & 48.59 & 13.86 & 54.18 & 15.93 & -1.242 & 0.221 \\
\hline Symbol coding & 55.14 & 13.28 & 57.09 & 13.44 & -0.485 & 0.630 \\
\hline ToL & 18.14 & 2.64 & 17.95 & 2.30 & 0.244 & 0.809 \\
\hline Total score & 251.45 & 40.13 & 275.05 & 37.17 & -2.023 & 0.050 \\
\hline \multicolumn{7}{|l|}{ PANSS } \\
\hline Positive factor & 10.10 & 1.92 & - & - & - & - \\
\hline Negative factor & 15.20 & 2.50 & - & - & - & - \\
\hline Disorganized factor & 7.43 & 1.78 & - & - & - & - \\
\hline Excited factor & 8.05 & 1.12 & - & - & - & - \\
\hline Depressed factor & 8.14 & 1.31 & - & - & - & - \\
\hline
\end{tabular}

Note that most patients received more than one antipsychotic medication, and four patients received additional antidepressive medication. No mood stabilizers, anticonvulsants or sedatives were prescribed.

Moreover, to explore whether there are possible schizophrenia phenotypes, a k-means clustering analysis was used. This was done to identify patient clusters with common symptoms in 4dimensional space based on power, ITC, PANSS positive, and negative factor (Schear, 1987; Olbrich et al., 2012). To this end, time-frequency power and ITC were averaged over the clusters obtained in the group comparison and data were sorted into an increasing number of clusters (i.e., the number of subjects for which all data were available minus one). Afterward, the optimal number of clusters was identified using the Akaike Information Criterion (AIC). Correlation and k-means cluster analyses were performed using R (R Development Core Team, 2011).

\section{RESULTS}

\section{Stimulus-Induced Oscillatory Power}

Auditory gating was defined as the difference in power increase following two paired auditory stimuli (i.e., S2-S1). The S2S1 difference between ScZ and HC was statistically assessed using independent-samples $t$-tests. In both groups, the S2 
(mean \pm SD, ScZ: $0.21 \pm 0.11$, HC: $0.30 \pm 0.22$ ) induced weaker power responses than the S1 (ScZ: $0.34 \pm 0.26$, HC: $0.63 \pm 0.51$ ), leading to negative $\mathrm{S} 2-\mathrm{S} 1$ gating differences. The gamma-band power gating difference at a mediocentral electrode cluster was significantly stronger in $\mathrm{HC}$ than in ScZ $[30-50 \mathrm{~Hz}, t(42)=2.37, p<0.05$, Figures 1A-E]. Additional ANOVAs and $t$-tests indicated that gamma-band power was not significantly different between ScZ and HC following S1 or S2 (see Supplementary Material). The S2-S1 gamma-band power difference was distributed normally ( $W=0.96, p=0.55)$. Within ScZ the gamma-band power gating difference correlated significantly with the PANSS positive factor $[r(19)=0.62$, $p<0.01$, Figure 1F]. The correlation remained significant when controlling for medication $[r(18)=0.57, p<0.01]$. Tables 2 and 3 summarize the main findings.

\section{Inter-Trial Coherence}

Sensory stimulation leads to a phase alignment shortly after stimulus onset (Cheron et al., 2007; Busch et al., 2009). Identical to the analysis of oscillatory power, the S2-S1 ITC was compared between ScZ and HC. In ScZ and HC, S2 (ScZ: $0.17 \pm 0.04, \mathrm{HC}$ : $0.19 \pm 0.06)$ induced a weaker ITC than S1 (ScZ: $0.22 \pm 0.05$,
A

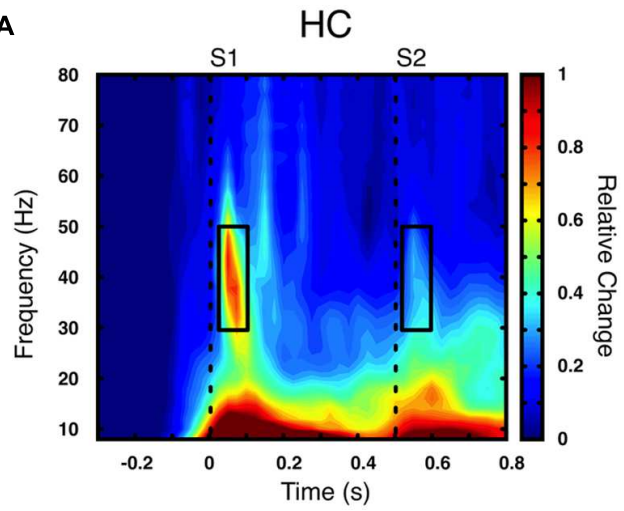

C

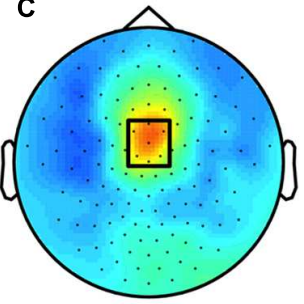

S1

$\begin{array}{lllllllll}0 & 0.1 & 0.2 & 0.3 & 0.4 & 0.5 & 0.6 & 0.7 & 0.8\end{array}$ Relative Change

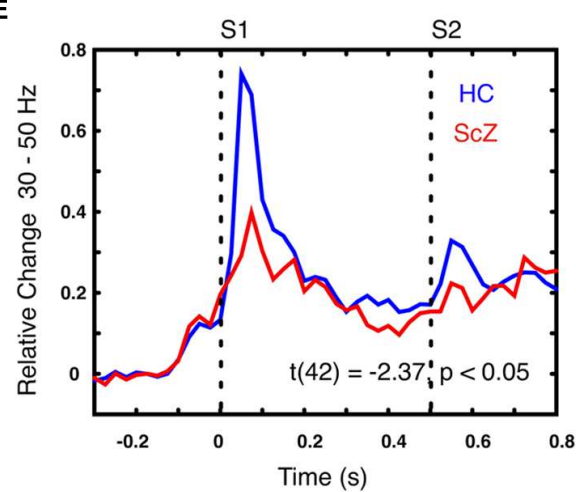

S2

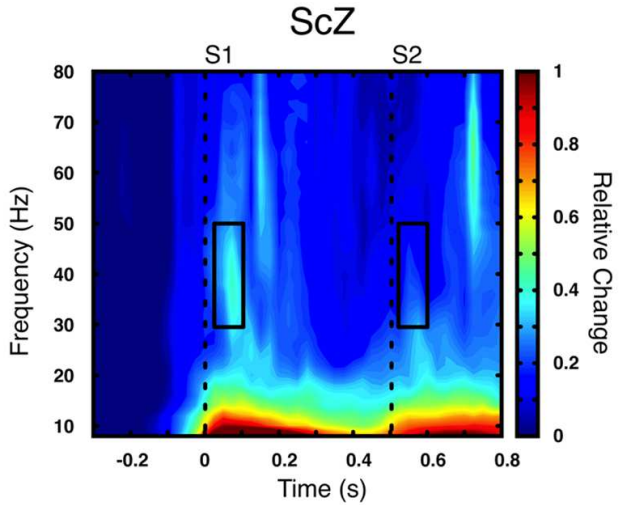

D
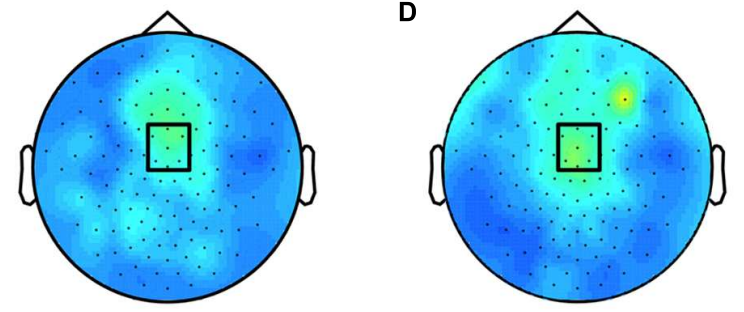

S1

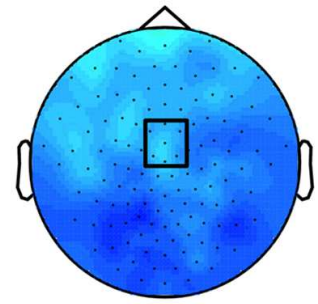

S2

$\begin{array}{lllllllll}0 & 0.1 & 0.2 & 0.3 & 0.4 & 0.5 & 0.6 & 0.7 & 0.8\end{array}$ Relative Change

$\mathbf{F}$

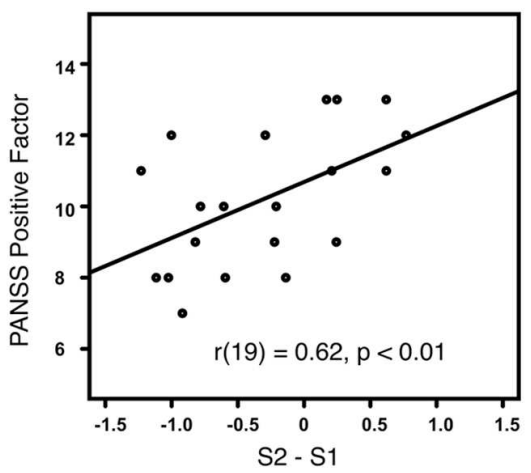

FIGURE 1 | Time-frequency representation of oscillatory power induced by the paired S1-S2 stimuli for HC and ScZ. (A,B) Depict changes in oscillatory power relative to baseline in mediocentral electrodes for $\mathrm{HC}$ and ScZ, respectively. Dashed lines indicate the onset of the auditory stimuli. Black boxes mark the time-frequency cluster $(0.05-0.1 \mathrm{~s} ; 30-50 \mathrm{~Hz})$ in which a significant auditory gating difference was found between groups. (C,D) Depict the topography of S1 and S2 for the time-frequency window marked in (A,B). Black boxes mark the mediocentral EEG electrode cluster, in which a significant auditory gating difference was found between groups depicted in (A,B). (E) Illustrates the time-course of mediocentral 30-50 Hz frequency window for HC (blue) and ScZ (red). (F) Illustrates the significant positive correlation between auditory gating in gamma-band power and the PANSS positive factor for ScZ. 
TABLE 2 | Overview of power and ITC effects.

\begin{tabular}{|c|c|c|c|c|c|c|c|}
\hline Metric & Stimulus & ScZ Mean & ScZ SD & HC Mean & HC SD & $t$-value & $p$-value \\
\hline Gamma-Band & S1 & 0.34 & 0.26 & 0.63 & 0.51 & 2.38 & $<0.05$ \\
\hline Power & S2 & 0.21 & 0.11 & 0.30 & 0.22 & 1.78 & 0.08 \\
\hline$(30-50 \mathrm{~Hz})$ & S2-S1 & -0.37 & 0.72 & -0.95 & 0.87 & 2.37 & $<0.05$ \\
\hline Alpha-Band & $\mathrm{S} 1$ & 0.22 & 0.05 & 0.33 & 0.10 & 4.25 & $<0.001$ \\
\hline ITC & $\mathrm{S} 2$ & 0.17 & 0.04 & 0.19 & 0.06 & 1.08 & 0.29 \\
\hline (8-12 Hz) & S2-S1 & -0.08 & 0.09 & -0.19 & 0.13 & 3.24 & $<0.01$ \\
\hline
\end{tabular}

Mean and SD values were computed over the sensor-time-frequency clusters obtained from the group comparison.

\begin{tabular}{|c|c|c|c|}
\hline Metric & PANSS-factors & $r(19)$ & $p$ \\
\hline Gamma-Band Power & Positive & 0.62 & $<0.005$ \\
\hline \multirow[t]{4}{*}{$(30-50 \mathrm{~Hz})$} & Negative & -0.27 & 0.24 \\
\hline & Disorganized & -0.23 & 0.31 \\
\hline & Excited & 0.14 & 0.55 \\
\hline & Depressed & 0.16 & 0.48 \\
\hline Alpha-Band ITC & Positive & 0.25 & 0.27 \\
\hline \multirow[t]{4}{*}{$(8-12 \mathrm{~Hz})$} & Negative & -0.54 & $<0.01$ \\
\hline & Disorganized & -0.37 & 0.09 \\
\hline & Excited & 0.04 & 0.83 \\
\hline & Depressed & 0.26 & 0.25 \\
\hline
\end{tabular}

Power and ITC values entered into the correlations were computed over the sensortime-frequency clusters obtained from the group comparison.

HC: $0.33 \pm 0.10$ ), leading to a negative ITC gating difference. The auditory gating difference was stronger in $\mathrm{HC}$ than in $\mathrm{ScZ}$, albeit in the alpha-band $[8-12 \mathrm{~Hz}, t(42)=3.24, p<0.01$, Figures 2A-E] in a mediocentral electrode cluster. Notably, this cluster was similar to the cluster obtained in the analysis of oscillatory power. Therefore, we chose the overlap between the clusters obtained in the analysis of oscillatory power and ITC to visualize the effects within one common region. Additional analyses revealed that alpha-band ITC was significantly stronger for HC than ScZ following S1 but not S2 (see Supplementary Material). The S2-S1 alpha-band ITC difference was distributed normally $(W=0.93, p=0.14$ ). In ScZ the individual alpha-band ITC gating difference was negatively correlated with the PANSS negative factor $[r(19)=-0.54, p<0.01$, Figure 2F]. Power modulations might have contributed to this relationship (see Supplementary Material), but importantly, the correlation remained significant when controlling for medication $[r(18)=-0.55, p<0.05]$. Tables 2 and 3 summarize the main findings.

\section{Symptom Clusters}

The gamma-band power gating difference was positively correlated with the alpha-band ITC gating difference in HC $[r(18)=0.62, p<0.01]$ and ScZ $[r(19)=0.46, p<0.05$, Figure 3A]. Within $S c Z$, the correlation remained significant when controlling for medication $[r(18)=0.51, p<0.05]$. Using the two electrophysiological markers (i.e., gamma-band power gating difference and alpha-band ITC gating difference) and the two PANSS factors (i.e., positive and negative), which were identified in the analyses above, a k-means cluster analysis was performed to identify schizophrenia phenotypes. The fourdimensional space (gamma-band power gating difference, alphaband ITC gating difference, PANSS positive factor scores, PANSS negative factor scores) was sorted into an increasing number of clusters. The AIC identified the three-cluster solution as optimal (Figure 3B). Note that the values of the four markers were transformed into $z$-scores. Hence, positive values indicate aberrant sensory gating and high symptom scores. Patients in the first cluster $(N=7)$ exhibited no sensory gating (i.e., gammaband power and alpha-band ITC to the S1 and S2 was similar), and strong positive, but low negative symptoms (Figure 3B, red trace). Patients in the second cluster $(N=6)$ were characterized by intact sensory gating in gamma-band power, but aberrant sensory gating in alpha-band ITC, and relatively low symptom scores (Figure 3B, black trace). Finally, patients in the third cluster $(N=8)$ expressed intact sensory gating in gamma-band power and alpha-band ITC, low positive symptoms but severe negative symptoms (Figure 3B, green trace).

\section{DISCUSSION}

Our study revealed that auditory sensory gating deficits relate to the schizophrenia psychopathology. While previous studies have also reported reduced sensory gating ratios in the P50, gamma-band power and alpha-band ITC (Clementz et al., 1997a; Brockhaus-Dumke et al., 2008; Patterson et al., 2008; Hall et al., 2011), a key novel finding of our study is that aberrant auditory sensory gating in gamma-band power correlates with positive symptoms in schizophrenia. Moreover, our study shows that aberrant temporal synchronization, as reflected in alpha-band phase consistency, correlates with negative symptoms. Using a cluster analysis approach, we identified three patient subgroups with distinct phenotypes of symptoms, and distinct alterations in gamma-band power and alpha-band ITC gating differences. In contrast to previous studies, we did not find differences between ScZ and HC in the auditory evoked P50 (see Supplementary Material).

\section{Positive Symptoms Correlate with Aberrant Gamma-Band Power Sensory Gating}

In line with the literature we found that schizophrenia patients exhibit an auditory sensory gating deficit in gamma-band power 

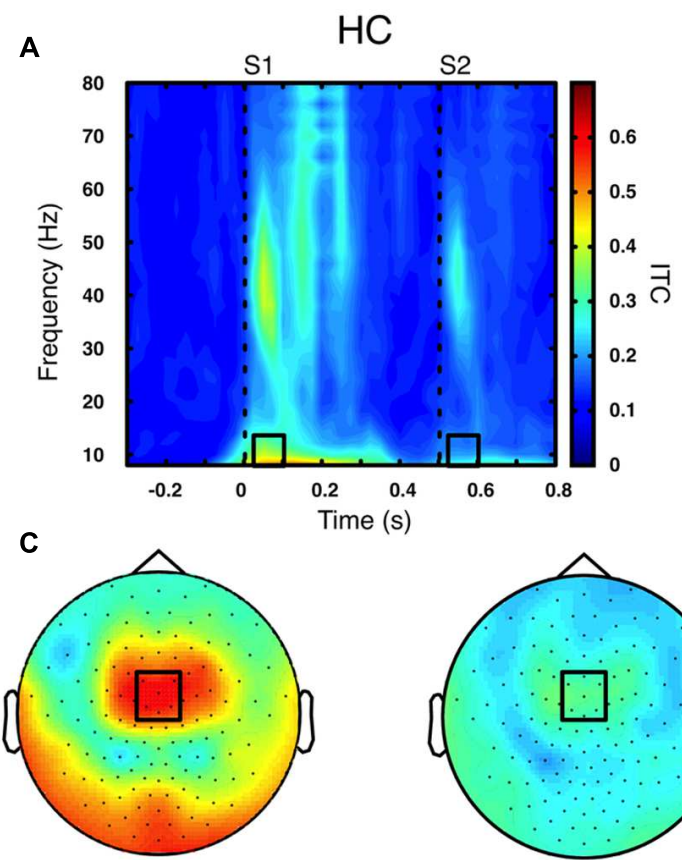

S1

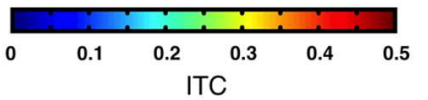

E

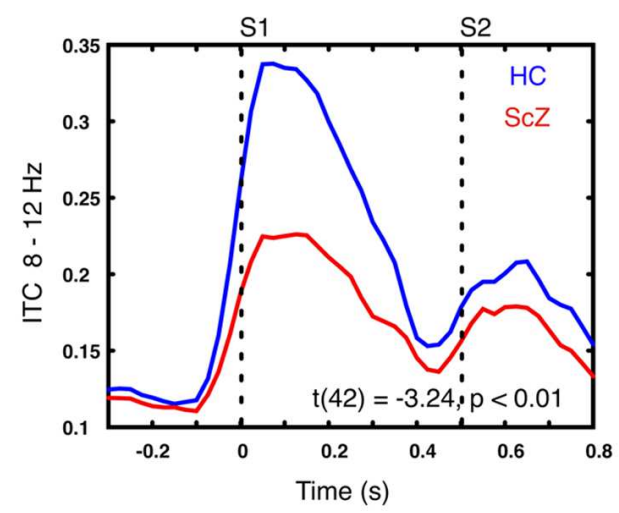

B
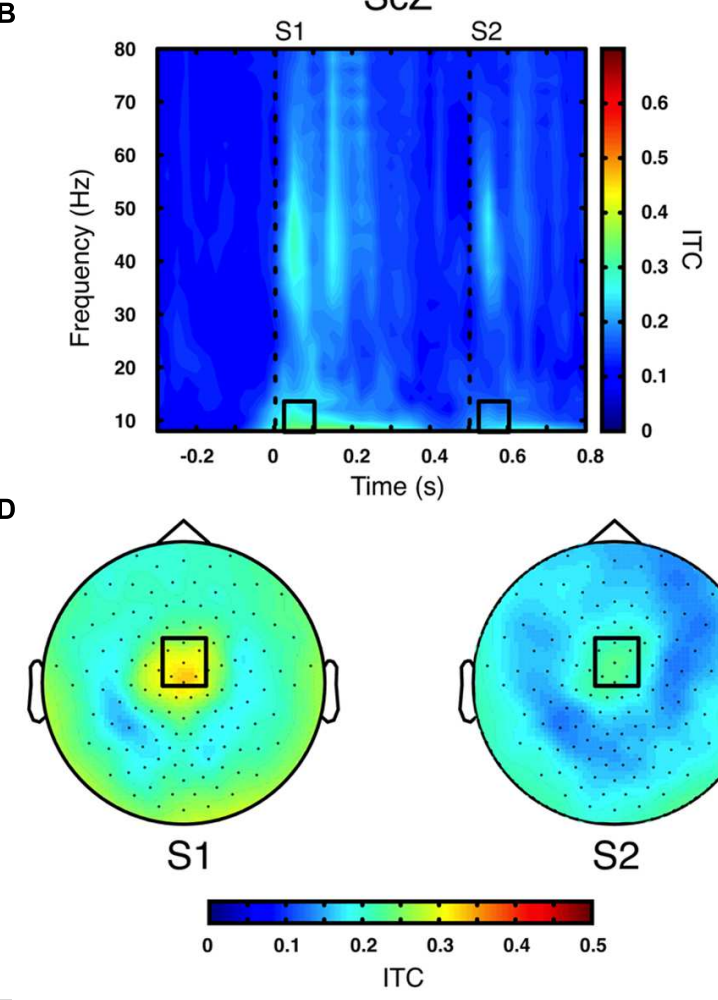

F

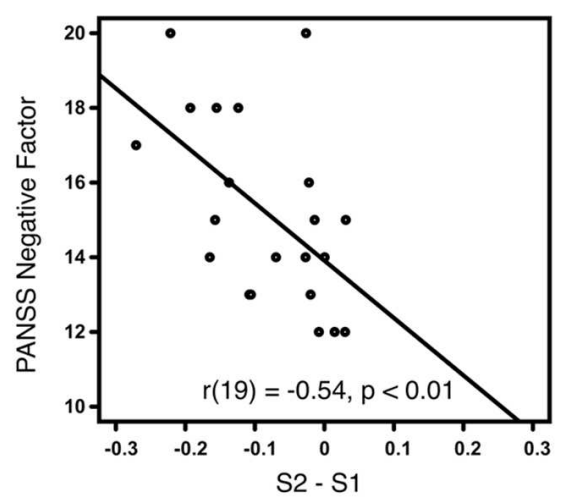

FIGURE 2 | Time-frequency representation of inter-trial coherence (ITC) induced by the paired S1-S2 stimuli for HC and ScZ. (A,B) Depict changes in ITC in mediocentral electrodes for $\mathrm{HC}$ and ScZ, respectively. Dashed lines indicate the onset of the auditory stimuli. Black boxes mark the time-frequency cluster $(0.05-0.1 \mathrm{~s} ; 8-12 \mathrm{~Hz}$ ) in which a significant auditory gating difference was found between groups. (C,D) Depict the topography of S1 and S2 for the time-frequency window marked in $\mathbf{( A , B ) . ~ B l a c k ~ b o x e s ~ m a r k ~ t h e ~ m e d i o c e n t r a l ~ E E G ~ e l e c t r o d e ~ c l u s t e r , ~ i n ~ w h i c h ~ a ~ s i g n i f i c a n t ~ a u d i t o r y ~ g a t i n g ~ d i f f e r e n c e ~ w a s ~ f o u n d ~ b e t w e e n ~ g r o u p s ~}$ depicted in (A,B). (E) Illustrates the time-course of mediocentral 8-12 Hz frequency window for HC (blue) and ScZ (red). (F) Illustrates the significant negative correlation between auditory gating in alpha-band ITC and the PANSS negative factor for ScZ.

(Clementz et al., 1997a; Hall et al., 2011; Popov et al., 2011, 2012). Extending previous findings, we observed that ScZ with the highest sensory gating deficits had the most severe positive symptoms. In line with our results, reduced gammaband amplitude and impaired gamma-band sensory gating has been associated with higher perceptual anomaly (Johannesen et al., 2008). In a similar vein, previous studies reported positive relationships between gamma-band power deficits and positive symptoms in other experimental paradigms, such as the auditory oddball paradigm (Lee et al., 2003b) or visual feature binding (Lee et al., 2003a; Spencer et al., 2004). Already half a century ago, it has been proposed that sensory gating deficits could lead to an information overload that contributes to the positive symptoms in schizophrenia (McGhie and Chapman, 1961; Shakow, 1963; Freedman et al., 1991; Bob et al., 2014). In agreement with this proposal, a recent study found that auditory sensory gating deficits relate to the severity of hallucinations in schizophrenia (Smith et al., 2013). Recently, Uhlhaas and 

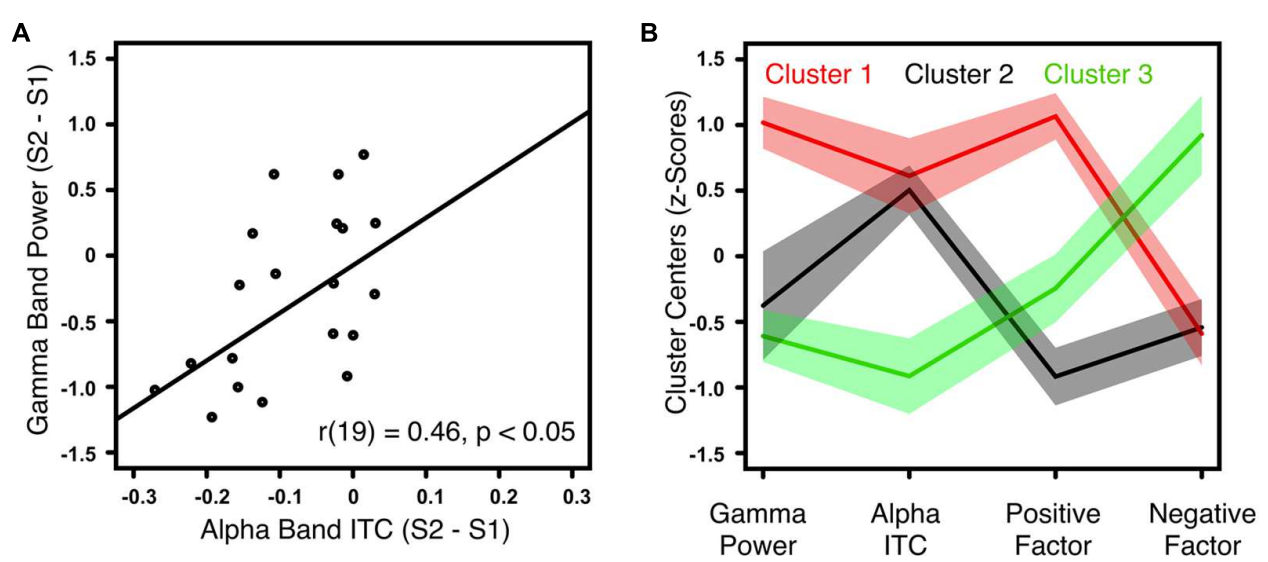

FIGURE 3 | Correlation between gamma-band power and alpha-band ITC for ScZ and outcome of the k-means cluster analysis. (A) IIlustrates the positive correlation between auditory gating (i.e., S2-S1) differences of induced gamma-band power and alpha-band ITC for ScZ. (B) Summarizes the symptom patterns of the three patient clusters identified by k-means clustering analysis. Positive values indicate aberrant sensory gating and high symptom scores. Negative values indicate intact sensory gating and low symptom scores. Solid lines mark the cluster centers, shaded areas mark the SEM.

Singer (2015) proposed that positive symptoms are secondary phenomena resulting from the attempt to cope with aberrant neural synchronization, as reflected in reduced gamma-band power. Thus, aberrant neural synchronization could lead to an inability to ignore irrelevant information, which in turn could contribute to positive symptoms. Coordinated oscillatory activity in neural populations is an important process underlying cognition (Engel et al., 2001; Senkowski et al., 2008; Wang, 2010; Pittman-Polletta et al., 2015). It has been shown that GABAergic inhibition to Parvalbumin-positive $(\mathrm{PV}+)$ interneurons is a key element in establishing gamma-band synchrony, as it controls when neural firing takes place (Royer et al., 2012). In schizophrenia, compromised functionality of $\mathrm{PV}+$ interneurons might lead to reduced gamma-band power and dysfunctional exaggerated locking of gamma-band oscillations to the trough of alpha-band oscillations (Popov and Popova, 2015).

Taken together, our study supports our hypothesis that gamma-band power gating deficits relate to positive symptoms in schizophrenia. The reduced gating in the induced gammaband power presumably reflects the inability to inhibit irrelevant sensory information.

\section{Negative Symptoms are Negatively Correlated with Aberrant Alpha-Band Inter-Trial Coherence Sensory Gating}

Negative symptoms are often found already in the prodromal phase of schizophrenia (Uhlhaas and Singer, 2015). These symptoms have been related to disorganized temporal synchronization (Andreasen et al., 1999). In line with this account, and in agreement with other reports (Leicht et al., 2010; Koh et al., 2011; Martin et al., 2013; Popov and Popova, 2015), we found diminished temporal synchronization as reflected in reduced phase coherence across trials in ScZ. Similar to the findings of altered gamma-band power, patients exhibited deficits in alpha-band ITC sensory gating. As previous studies have reported a reduction of alpha-band ITC (Jansen et al., 2004; Brockhaus-Dumke et al., 2008), it is somewhat surprising that schizophrenia patients who showed stronger sensory gating reported more severe negative symptoms. Previous studies have reported that negative symptoms, low levels of functioning, and reduced processing speeds are related to reduced alpha- and beta-band power (Hong et al., 2012; Smucny et al., 2013). The negative correlation between sensory gating and negative symptoms in the present study might indicate that these symptoms are related to cognitive dysfunctions and not primarily related to altered sensory processing. Our finding also seems to be in contrast with two previous studies, which reported that aberrant P50 sensory gating relates to more severe negative symptoms (Ringel et al., 2004; Thoma et al., 2005). However, these previous studies investigated the broadband evoked signal. In the present study, we analyzed the frequency-resolved signal. Hence, our finding of an alpha-band ITC gating deficit is in line with our hypothesis of reduced temporal synchronization in schizophrenia. In the prodromal phase, negative symptoms are often already present, and it is possible that altered alpha-band activity reflects a functional decline before the actual onset of the psychosis (Koh et al., 2011; Fusar-Poli et al., 2013). Interestingly, alpha-band ITC was significantly decreased in ScZ following S1. This suggests that schizophrenia patients not only suffer from an inability to inhibit irrelevant information, but also exhibit a stimulus-encoding deficit (Mathiak et al., 2011; Popov et al., 2011).

\section{Cluster Analysis Reveals Schizophrenia Phenotype Subgroups}

An important goal in psychiatric research is to identify distinct phenotypes within the population of patients (Andreasen, 2000; Smucny et al., 2013; Boutros et al., 2014). Relating different measures of sensory gating to clinical parameters, as done in our study, can provide novel insight into the aberrant cortical processes that contribute to the schizophrenia psychopathology. 
To uncover possible phenotypes of schizophrenia, clinical, and cortical parameters have to be integrated. Using $\mathrm{k}$-means clustering, we identified ScZ clusters with distinct deficits in oscillatory processes, and clinical symptoms. In line with the notion that positive symptoms relate to aberrant sensory gating (McGhie and Chapman, 1961; Shakow, 1963), we found one patient cluster that was characterized by a sensory gating deficit in gamma-band power and alpha-band ITC. These patients also showed pronounced positive symptoms. A second patient cluster was characterized by low positive and negative symptom scores, and intact sensory gating in the gamma-band, but aberrant gating in alpha-band ITC. Patients with aberrant temporal synchronization might suffer from cognitive deficits not limited to positive or negative symptoms (Andreasen et al., 1999; Popov and Popova, 2015). Finally, patients in the third cluster showed strong negative symptoms but relatively intact sensory gating in the alpha- and gamma-band. Negative symptoms in these patients might thus be less related to sensory gating but rather to other cognitive deficits, such as poor attention or disorientation. It is important to note that due to the low number of participants, the cluster analysis approach has an explorative character. Thus, the outcome of our analysis should be verified in a larger sample. It is also possible that group differences and correlations may be influenced by the effect of pharmacological agents on sensory gating. However, all correlations remained significant after controlling for medication. Taken together, the results of the cluster analysis underline the relationship between abnormal sensory gating in gamma-band power and positive symptoms. Conversely, abnormal gating in alpha-band ITC might relate more to unspecific symptoms in ScZ (Koh et al., 2011; FusarPoli et al., 2013). Our study provides novel insight into the multifaceted relationships between aberrant neural oscillations and schizophrenia psychopathology. Cluster based approaches, which integrate clinical symptoms and neural activity, seem to be suitable to characterize these relationships on an individual level.

\section{SUMMARY}

We related oscillatory power and temporal synchronization of time-frequency resolved EEG data to positive and negative

\section{REFERENCES}

Adler, L. E., Pachtman, E., Franks, R. D., Pecevich, M., Waldo, M. C., and Freedman, R. (1982). Neurophysiological evidence for a defect in neuronal mechanisms involved in sensory gating in schizophrenia. Biol. Psychiatry 17, 639-654.

Andreasen, N. C. (2000). Schizophrenia: the fundamental questions. Brain Res. Brain Res. Rev. 31, 106-112. doi: 10.1016/S0165-0173(99) 00027-2

Andreasen, N. C., Nopoulos, P., O’Leary, D. S., Miller, D. D., Wassink, T., and Flaum, M. (1999). Defining the phenotype of schizophrenia: cognitive dysmetria and its neural mechanisms. Biol. Psychiatry 46, 908-920. doi: 10.1016/S0006-3223(99)00152-3

Bob, P., Vlcek, P., and Raboch, J. (2014). Sensory disturbances, inhibitory deficits, and the P50 wave in schizophrenia. Neuropsychiatr. Dis. Treat. 10, 1309-1315. doi: $10.2147 /$ NDT.S64219 symptoms in schizophrenia. Our study shows that the sensory gating deficit in oscillatory power is positively correlated with the positive symptoms in schizophrenia. Moreover, the sensory gating deficit in temporal synchronization is negatively correlated with negative symptoms. In addition, our cluster analysis uncovered distinct schizophrenia phenotypes. We identified three patient clusters defined by common patterns of sensory gating deficits in gamma- and alpha-band oscillations, as well as similarities in positive and negative symptoms. This shows that examining oscillatory activity enables to uncover distinct schizophrenia phenotypes. Conclusively, our study suggests that analyzing different aspects of oscillatory activity in larger cohorts, and defining specific phenotypes will substantially further our understanding of the schizophrenia psychopathology.

\section{AUTHOR CONTRIBUTIONS}

JK, YRR, JB, and DS designed the experiment. YRR, JB, and MH recorded the data. JK, YRR, and $\mathrm{MH}$ analyzed the data. JK, YRR, $\mathrm{JB}, \mathrm{MH}$, and DS wrote the manuscript.

\section{FUNDING}

This work was supported by the European Union (ERC-2010StG-20091209 to DS) and the German Research Foundation (SE1859/4-1 to DS and KE1828/2-1 to JK).

\section{ACKNOWLEDGMENTS}

We thank the volunteers, especially the patients, for participation in this study. We thank Markus Koch, Paulina Schulz, and Christiane Montag for their assistance.

\section{SUPPLEMENTARY MATERIAL}

The Supplementary Material for this article can be found online at: http://journal.frontiersin.org/article/10.3389/fnhum. 2016.00104

Boutros, N. N., Mucci, A., Diwadkar, V., and Tandon, R. (2014). Negative symptoms in schizophrenia: a comprehensive review of electrophysiological investigations. Clin. Schizophr. Relat. Psychosis 8, 28-35. doi: 10.3371/CSRP.BOMU.012513

Bramon, E., Rabe-Hesketh, S., Sham, P., Murray, R. M., and Frangou, S. (2004). Meta-analysis of the P300 and P50 waveforms in schizophrenia. Schizophr. Res. 70, 315-329. doi: 10.1016/j.schres.2004. 01.004

Brenner, C. A., Kieffaber, P. D., Clementz, B. A., Johannesen, J. K., Shekhar, A., O'Donnell, B. F., et al. (2009). Event-related potential abnormalities in schizophrenia: a failure to "gate in" salient information? Schizophr. Res. 113, 332-338. doi: 10.1016/j.schres.2009.06.012

Brockhaus-Dumke, A., Mueller, R., Faigle, U., and Klosterkoetter, J. (2008). Sensory gating revisited: relation between brain oscillations and auditory evoked potentials in schizophrenia. Schizophr. Res. 99, 238-249. doi: 10.1016/j.schres.2007.10.034 
Busch, N. A., Dubois, J., and Vanrullen, R. (2009). The phase of ongoing EEG oscillations predicts visual perception. J. Neurosci. 29, 7869-7876. doi: 10.1523/JNEUROSCI.0113-09.2009

Carolus, A. M., Schubring, D., Popov, T. G., Popova, P., Miller, G. A., and Rockstroh, B. S. (2014). Functional cognitive and cortical abnormalities in chronic and first-admission schizophrenia. Schizophr. Res. 157, 40-47. doi: 10.1016/j.schres.2014.05.012

Chaumon, M., Bishop, D. V. M., and Busch, N. A. (2015). A practical guide to the selection of independent components of the electroencephalogram for artifact correction. J. Neurosci. Methods 250, 47-63. doi: 10.1016/j.jneumeth.2015.02.025

Cheron, G., Cebolla, A., De Saedeleer, C., Bengoetxea, A., Leurs, F., Leroy, A., et al. (2007). Pure phase-locking of beta/gamma oscillation contributes to the N30 frontal component of somatosensory evoked potentials. BMC Neurosci. 8:75. doi: 10.1186/1471-2202-8-75

Clementz, B. A., Blumenfeld, L. D., and Cobb, S. (1997a). The gamma band response may account for poor P50 suppression in schizophrenia. Neuroreport 8, 3889-3893. doi: 10.1097/00001756-199712220-00010

Clementz, B. A., Geyer, M. A., and Braff, D. L. (1997b). P50 suppression among schizophrenia and normal comparison subjects: a methodological analysis. Biol. Psychiatry 41, 1035-1044. doi: 10.1016/S0006-3223(96)00208-9

Delorme, A., and Makeig, S. (2004). EEGLAB: an open source toolbox for analysis of single-trial EEG dynamics including independent component analysis. J. Neurosci. Methods 134, 9-21. doi: 10.1016/j.jneumeth.2003. 10.009

Engel, A. K., Fries, P., and Singer, W. (2001). Dynamic predictions: oscillations and synchrony in top-down processing. Nat. Rev. Neurosci. 2, 704-716. doi: $10.1038 / 35094565$

Ethridge, L. E., Hamm, J. P., Pearlson, G. D., Tamminga, C. A., Sweeney, J. A., Keshavan, M. S., et al. (2015). Event-related potential and timefrequency endophenotypes for schizophrenia and psychotic bipolar disorder. Biol. Psychiatry 77, 127-136. doi: 10.1016/j.biopsych.2014.03.032

Freedman, R., Waldo, M., Bickford-Wimer, P., and Nagamoto, H. (1991). Elementary neuronal dysfunctions in schizophrenia. Schizophr. Res. 4, 233-243. doi: 10.1016/0920-9964(91)90035-P

Fusar-Poli, P., Borgwardt, S., Bechdolf, A., Addington, J., Riecher-Rössler, A., Schultze-Lutter, F., et al. (2013). The psychosis high-risk state. JAMA Psychiatry 70, 107. doi: 10.1001/jamapsychiatry.2013.269

Gallinat, J., Winterer, G., Herrmann, C. S., and Senkowski, D. (2004). Reduced oscillatory gamma-band responses in unmedicated schizophrenic patients indicate impaired frontal network processing. Clin. Neurophysiol. 115, 18631874. doi: 10.1016/j.clinph.2004.03.013

Gardner, D. M., Murphy, A. L., O’Donnell, H., Centorrino, F., and Baldessarini, R. J. (2010). International consensus study of antipsychotic dosing. Am. J. Psychiatry 167, 686-693. doi: 10.1176/appi.ajp.2009.09060802

Hall, M. H., Taylor, G., Salisbury, D. F., and Levy, D. L. (2011). Sensory gating event-related potentials and oscillations in schizophrenia patients and their unaffected relatives. Schizophr. Bull. 37, 1187-1199. doi: 10.1093/schbul/sbq027

Hong, L. E., Summerfelt, A., Mitchell, B. D., O’Donnell, P., and Thaker, G. K. (2012). A shared low-frequency oscillatory rhythm abnormality in resting and sensory gating in schizophrenia. Clin. Neurophysiol. 123, 285-292. doi: 10.1016/j.clinph.2011.07.025

Jansen, B. H., Hegde, A., and Boutros, N. N. (2004). Contribution of different EEG frequencies to auditory evoked potential abnormalities in schizophrenia. Clin. Neurophysiol. 115, 523-533. doi: 10.1016/j.clinph.2003.10.016

Johannesen, J. K., Bodkins, M., O'Donnell, B. F., Shekhar, A., and Hetrick, W. P. (2008). Perceptual anomalies in schizophrenia co-occur with selective impairments in the gamma frequency component of midlatency auditory ERPs. J. Abnorm. Psychol. 117, 106-118. doi: 10.1037/0021-843X.117.1.106

Kay, S. R., Fiszbein, A., and Opler, L. A. (1987). The positive and negative syndrome scale (PANSS) for schizophrenia. Schizophr. Bull. 13, 261-276. doi: $10.1093 / \mathrm{schbul} / 13.2 .261$

Keefe, R. (2004). The Brief Assessment of Cognition in Schizophrenia: reliability, sensitivity, and comparison with a standard neurocognitive battery. Schizophr. Res. 68, 283-297. doi: 10.1016/j.schres.2003.09.011

Keshavan, M. S., Tandon, R., Boutros, N. N., and Nasrallah, H. A. (2008). Schizophrenia, "just the facts": what we know in 2008 Part 3: neurobiology. Schizophr. Res. 106, 89-107. doi: 10.1016/j.schres.2008.07.020
Koh, Y., Shin, K. S., Kim, J. S., Choi, J.-S., Kang, D.-H., Jang, J. H., et al. (2011). An MEG study of alpha modulation in patients with schizophrenia and in subjects at high risk of developing psychosis. Schizophr. Res. 126, 36-42. doi: 10.1016/j.schres.2010.10.001

Lee, K.-H., Williams, L. M., Breakspear, M., and Gordon, E. (2003a). Synchronous Gamma activity: a review and contribution to an integrative neuroscience model of schizophrenia. Brain Res. Rev. 41, 57-78. doi: 10.1016/S00068993(02)03841-6

Lee, K.-H., Williams, L. M., Haig, A., and Gordon, E. (2003b). "Gamma (40 $\mathrm{Hz}$ ) phase synchronicity" and symptom dimensions in schizophrenia. Cogn. Neuropsychiatry 8, 57-71. doi: 10.1080/713752240

Lee, T. W., Girolami, M., and Sejnowski, T. J. (1999). Independent component analysis using an extended infomax algorithm for mixed subgaussian and supergaussian sources. Neural Comput. 11, 417-441. doi: $10.1162 / 089976699300016719$

Leicht, G., Kirsch, V., Giegling, I., Karch, S., Hantschk, I., Möller, H. J., et al. (2010). Reduced early auditory evoked gamma-band response in patients with schizophrenia. Biol. Psychiatry 67, 224-231. doi: 10.1016/j.biopsych.2009.07.033

Lisman, J. (2012). Excitation, inhibition, local oscillations, or large-scale loops: what causes the symptoms of schizophrenia? Curr. Opin. Neurobiol. 22, 537544. doi: 10.1016/j.conb.2011.10.018

Louchart-de la Chapelle, S., Levillain, D., Ménard, J.-F., Van der Elst, A., Allio, G., Haouzir, S., et al. (2005). P50 inhibitory gating deficit is correlated with the negative symptomatology of schizophrenia. Psychiatry Res. 136, 27-34. doi: 10.1016/j.psychres.2003.04.001

Maris, E., and Oostenveld, R. (2007). Nonparametric statistical testing of EEG- and MEG-data. J. Neurosci. Methods 164, 177-190. doi: 10.1016/j.jneumeth.2007.03.024

Martin, B., Giersch, A., Huron, C., and van Wassenhove, V. (2013). Temporal event structure and timing in schizophrenia: preserved binding in a longer "now". Neuropsychologia 51, 358-371. doi: 10.1016/j.neuropsychologia.2012. 07.002

Mathiak, K., Ackermann, H., Rapp, A., Mathiak, K. A., Shergill, S., Riecker, A., et al. (2011). Neuromagnetic oscillations and hemodynamic correlates of P50 suppression in schizophrenia. Psychiatry Res. 194, 95-104. doi: 10.1016/j.pscychresns.2011.01.001

McGhie, A., and Chapman, J. (1961). Disorders of attention and perception in early schizophrenia. Br. J. Med. Psychol. 34, 103-116. doi: 10.1111/j.20448341.1961.tb00936.x

Mitra, P. P., and Pesaran, B. (1999). Analysis of dynamic brain imaging data. Biophys. J. 76, 691-708. doi: 10.1016/S0006-3495(99)77236-X

Moran, Z. D., Williams, T. J., Bachman, P., Nuechterlein, K. H., Subotnik, K. L., and Yee, C. M. (2012). Spectral decomposition of P50 suppression in schizophrenia during concurrent visual processing. Schizophr. Res. 140, 237-242. doi: 10.1016/j.schres.2012.07.002

Olbrich, S., Sander, C., Minkwitz, J., Chittka, T., Mergl, R., Hegerl, U., et al. (2012). EEG vigilance regulation patterns and their discriminative power to separate patients with major depression from healthy controls. Neuropsychobiology 65, 188-194. doi: 10.1159/000337000

Oostenveld, R., Fries, P., Maris, E., and Schoffelen, J.-M. (2011). FieldTrip: open source software for advanced analysis of MEG, EEG, and invasive electrophysiological data. Comput. Intell. Neurosci. 2011, 1-9. doi: $10.1155 / 2011 / 156869$

Pantev, C., Makeig, S., Hoke, M., Galambos, R., Hampson, S., and Gallen, C. (1991). Human auditory evoked gamma-band magnetic fields. Proc. Natl. Acad. Sci. U.S.A. 88, 8996-9000. doi: 10.1073/pnas.88.20.8996

Park, H. R. P., Lim, V. K., Kirk, I. J., and Waldie, K. E. (2015). P50 sensory gating deficits in schizotypy. Pers. Individ. Dif. 82, 142-147. doi: 10.1016/j.paid.2015.03.025

Patterson, J. V., Hetrick, W. P., Boutros, N. N., Jin, Y., Sandman, C., Stern, H., et al. (2008). P50 sensory gating ratios in schizophrenics and controls: a review and data analysis. Psychiatry Res. 158, 226-247. doi: 10.1016/j.psychres.2007.02.009

Pittman-Polletta, B. R., Kocsis, B., Vijayan, S., Whittington, M. A., and Kopell, N. J. (2015). Brain rhythms connect impaired inhibition to altered cognition in schizophrenia. Biol. Psychiatry 77, 1020-1030. doi: 10.1016/j.biopsych.2015.02.005

Popov, T., Jordanov, T., Weisz, N., Elbert, T., Rockstroh, B., and Miller, G. A. (2011). Evoked and induced oscillatory activity contributes to abnormal 
auditory sensory gating in schizophrenia. Neuroimage 56, 307-314. doi: 10.1016/j.neuroimage.2011.02.016

Popov, T., and Popova, P. (2015). Same clock, different time read-out: spontaneous brain oscillations and their relationship to deficient coding of cognitive content. Neuroimage 119, 316-324. doi: 10.1016/j.neuroimage.2015. 06.071

Popov, T., Rockstroh, B., Weisz, N., Elbert, T., and Miller, G. A. (2012). Adjusting brain dynamics in schizophrenia by means of perceptual and cognitive training. PLoS ONE 7:e39051. doi: 10.1371/journal.pone.0039051.t001

Potter, D., Summerfelt, A., Gold, J., and Buchanan, R. W. (2005). Review of clinical correlates of P50 sensory gating abnormalities in patients with schizophrenia. Schizophr. Bull. 32, 692-700. doi: 10.1093/schbul/sbj050

R Development Core Team (2011). R: A Language and Environment for Statistical Computing. Vienna: R Foundation for Statistical Computing.

Reulbach, U., Bleich, S., Maih ouml fner, C., Kornhuber, J., and Sperling, W. (2007). Specific and unspecific auditory hallucinations in patients with schizophrenia. Neuropsychobiology 55, 89-95. doi: 10.1159/000103907

Ringel, T. M., Heidrich, A., Jacob, C. P., and Fallgatter, A. J. (2004). Sensory gating deficit in a subtype of chronic schizophrenic patients. Psychiatry Res. 125, 237-245. doi: 10.1016/j.psychres.2004.01.004

Royer, S., Zemelman, B. V., Losonczy, A., Kim, J., Chance, F., Magee, J. C., et al. (2012). Control of timing, rate and bursts of hippocampal place cells by dendritic and somatic inhibition. Nat. Neurosci. 15, 769-775. doi: 10.1038/nn.3077

Schear, J. M. (1987). Utility of cluster analysis in classification of mixed neuropsychiatric patients. Arch. Clin. Neuropsychol. 2, 329-341. doi: 10.1016/0887-6177(87)90002-3

Senkowski, D., and Gallinat, J. (2015). Dysfunctional prefrontal gammaband oscillations reflect working memory and other cognitive deficits in schizophrenia. Biol. Psychiatry 77, 1010-1019. doi: 10.1016/j.biopsych.2015.02.034

Senkowski, D., Schneider, T., Foxe, J., and Engel, A. (2008). Crossmodal binding through neural coherence: implications for multisensory processing. Trends Neurosci. 31, 401-409. doi: 10.1016/j.tins.2008.05.002

Shakow, D. (1963). Psychological deficit in schizophrenia. Behav. Sci. 8, 275-305. doi: $10.1002 /$ bs.3830080402

Singer, W. (1999). Neuronal synchrony: a versatile code for the definition of relations? Neuron 24, 49-65. doi: 10.1016/S0896-6273(00)80821-1

Smith, D. M., Grant, B., Fisher, D. J., Borracci, G., Labelle, A., and Knott, V. J. (2013). Auditory verbal hallucinations in schizophrenia correlate with P50 gating. Clin. Neurophysiol. 124, 1329-1335. doi: 10.1016/j.clinph.2013. 02.004

Smucny, J., Wylie, K., Rojas, D., Stevens, K., Olincy, A., Kronberg, E., et al. (2013). Evidence for gamma and beta sensory gating deficits as translational endophenotypes for schizophrenia. Psychiatry Res. 214, 169-174. doi: 10.1016/j.pscychresns.2013.07.002

Spencer, K. M., Nestor, P. G., Perlmutter, R., Niznikiewicz, M. A., Klump, M. C., Frumin, M., et al. (2004). Neural synchrony indexes disordered perception and cognition in schizophrenia. Proc. Natl. Acad. Sci. U.S.A. 101, 17288-17293. doi: 10.1073/pnas.0406074101

Spencer, K. M., Niznikiewicz, M. A., Nestor, P. G., Shenton, M. E., and McCarley, R. W. (2009). Left auditory cortex gamma synchronization and auditory hallucination symptoms in schizophrenia. BMC Neurosci. 10:85. doi: 10.1186/1471-2202-10-85

Sponheim, S. R., Clementz, B. A., Iacono, W. G., and Beiser, M. (2000). Clinical and biological concomitants of resting state EEG power abnormalities in schizophrenia. Biol. Psychiatry 48, 1088-1097. doi: 10.1016/S00063223(00)00907-0

Thoma, R. J., Hanlon, F. M., Moses, S. N., Ricker, D., Huang, M., Edgar, C., et al. (2005). M50 sensory gating predicts negative symptoms in schizophrenia. Schizophr. Res. 73, 311-318. doi: 10.1016/j.schres.2004.07.001

Tiitinen, H., Sinkkonen, J., Reinikainen, K., Alho, K., Lavikainen, J., and Näätänen, R. (1993). Selective attention enhances the auditory 40-Hz transient response in humans. Nature 364, 59-60. doi: 10.1038/364059a0

Uhlhaas, P. J., and Singer, W. (2010). Abnormal neural oscillations and synchrony in schizophrenia. Nat. Rev. Neurosci. 11, 100-113. doi: 10.1038/nrn2774

Uhlhaas, P. J., and Singer, W. (2015). Oscillations and neuronal dynamics in schizophrenia: the search for basic symptoms and translational opportunities. Biol. Psychiatry 77, 1001-1009. doi: 10.1016/j.biopsych.2014.11.019

Wallwork, R. S., Fortgang, R., Hashimoto, R., Weinberger, D. R., and Dickinson, D. (2012). Searching for a consensus five-factor model of the Positive and Negative Syndrome Scale for schizophrenia. Schizophr. Res. 137, 246-250. doi: 10.1016/j.schres.2012.01.031

Wang, X.-J. (2010). Neurophysiological and computational principles of cortical rhythms in cognition. Physiol. Rev. 90, 1195-1268. doi: 10.1152/physrev.00035.2008

Conflict of Interest Statement: The authors declare that the research was conducted in the absence of any commercial or financial relationships that could be construed as a potential conflict of interest.

Copyright (C) 2016 Keil, Roa Romero, Balz, Henjes and Senkowski. This is an openaccess article distributed under the terms of the Creative Commons Attribution License (CC BY). The use, distribution or reproduction in other forums is permitted, provided the original author(s) or licensor are credited and that the original publication in this journal is cited, in accordance with accepted academic practice. No use, distribution or reproduction is permitted which does not comply with these terms. 